\title{
Editorial
}

\section{An unsatisfactory report card}

The web site http://www.cushings-help.com/ side_effects.htm made for some interesting and challenging reading. The first section reads: 'Side effects of steroid therapy considered acceptable and expected by the prescriber (though not by the patient!)'. That is quite an indictment against us as clinicians, but is it accurate? Regrettably, not only is the statement correct, but as evidenced elsewhere in this issue of the journal (1), the indictment does not go far enough.

McDougall et al. (1) report a 10 year retrospective chart review of 512 patients with neuromuscular disease of whom $209 \quad(41 \%)$ received corticosteroids at some point in their management. The diseases being treated varied widely as did the dose and duration of steroid therapy. The average dose $26.6 \mathrm{mg}$ /day and the average duration of therapy 4.5 years so it is hardly surprising that three out of every four patients receiving steroids experienced one or more adverse effects. About $29 \%$ of the patients experienced cosmetic changes such as acne or hirsutism, and $29 \%$ had an increase appetite and weight gain - effects that are allegedly considered acceptable and expected by the prescriber but not the patient. One might include psychological changes $(21 \%)$ in this category. To a certain extent this is excusable since there is little that can be offered either prophylactically or even therapeutically for these side effects. Fewer patients had diabetes $(12 \%)$ but checking for this life changing complication on an annual basis was only done in $38 \%$ of the patients. Guidelines for osteoporosis prophylaxis clearly state that this should be considered in all patients receiving steroids for 3 months or longer. Three quarters of the patients were on steroids for $>6$ months yet osteoporosis prophylaxis was only considered in about half of the patients.

Another specialty where use of steroids is common and appropriate is Rheumatology but there too attention to the adverse effects of chronic steroid therapy is apparently sub-optimal (2). About $60 \%$ of patients with rheumatoid arthritis were considered long-term users receiving $\geq 5 \mathrm{mg} /$ day for $\geq 6$ months. Of these only $37 \%$ received baseline bone density scans and in that group bone loss was documented in $70 \%$. Yet only $40 \%$ received either prophylaxis or therapy. Failure to appreciate and prevent complications from therapy is not restricted to chronic corticosteroid therapy. In particular adverse but preventable side effects of aromatase inhibitor and androgen deprivation therapies for breast and prostate cancer respectively are often ignored $(3,4)$.

These deficiencies in patient care are easy to understand as therapies such as steroids or hormone ablation are not prescribed indiscriminately but are offered to patients with serious diseases for which therapies free of major adverse events are simply not available. That is not offered as an excuse but as an urgent reminder for clinicians to develop and implement management algorithms that make it almost automatic to attend to these possible side effects within a few weeks of initiating long-term steroid therapy. Often steroids are initiated on a trial basis to see if any clinical improvement can be achieved and, when that does occur there is reluctance on the part of the physician and patient to discontinue. It would seem reasonable to evaluate each patient after 4 weeks of therapy and at that time become proactive concerning the long term consequences of therapy.

There are a number of published clinical guidelines relating to the protection of skeletal health in patients beginning long-term steroid therapy but none specifically related to neurologic disease. An osteoporosis-related fragility fracture is defined as a fracture following trauma equal to or less than a fall from a standing height. For patients with neurologic disease the operative word here is 'fall'! Any patient with neurologic disease that is associated with an increased tendency to fall should have an assessment of bone mineral density, whether they are or are not receiving steroid therapy. They should also be cautioned about fall prevention both inside the house (where many hip fractures occur) and outside the house. Those with low bone density and those on steroids should (probably independent of baseline bone density) be strongly considered for therapy with bisphosphonates.

Prophylaxis against diabetes is much more difficult, aggravated by the epidemic of obesity 
and the tough economic environment with appropriate nutrition getting beyond the reach of so many. Evidence-based guidelines are few and far between whether the patient is on steroids or not. Particular attention should be paid to those who are overweight or obese as determined by BMI, as should those with a positive family history of diabetes. It would seem reasonable and cost effective to consider obtaining a fasting blood glucose and hemoglobin A1C on every patient but a fasting blood test may not be practical. An alternative would be an in office measurement of capillary blood glucose (CBG) with a glucose meter (there is no need to fast for an A1C test). CBG could be measured at every visit and A1C monitored every 3 months. Those in whom previously unrecognized diabetes is uncovered as steroid therapy is begun should be started on oral medication. All patients starting long-term steroid therapy should be reminded about the increase appetite that this treatment causes and advised to be as careful as possible about what they eat. The evidence that this advice is effective is not very strong but that should not deter the clinician from giving such advice, since the patient is unlikely to get it elsewhere.

Adverse events associated with symptoms (gastro-intestinal, ocular, and dermatologic including hirsutism) cannot be prevented in most instances but they do require immediate and appropriate attention when noted by the patient. Cosmetic changes noted by the clinician but not mentioned by the patient should perhaps be handled a bit more circumspectly.

Adverse events complicating chronic steroid are frequent, often unavoidable, and difficult to manage once they occur. In most patients, the benefits of the therapy far outweigh the harm caused but it should be incumbent on all clinicians who prescribe long-term steroids to have a patient friendly guide clearly discussing the likely benefits and potential side effects, with an emphasis on the benefits. This will not stop the patient from looking for the side effect but mainly when the benefits are not obvious. If the benefits are not demonstrable to the patient with the history, physical examination or appropriate laboratory studies the decision whether to continue the steroids becomes paramount.

The literature support for this editorial is sparse. That is not for want of seeking adequate support for my comments - extensive searching for guidelines to cite was not fruitful. The guideline need not be evidenced based - there is nothing wrong with common sense. The report by McDougall et al. clearly documents what one needs to be on the lookout for, and every clinician caring for diseases that may require long-term steroid therapy can use that to develop a check list to place on the cover of the chart of every patient for whom they prescribe steroids.

PS: Another item to keep in your clinic for patients on long-term steroids - a brochure explaining how to obtain a Medic Alert dog-tag or bracelet alerting any emergency care giver to the ongoing or previous long-term steroid use.

M. Kleerekoper

St. Joseph Mercy Hospital, Ann Arbor, MI, USA and Department of Internal Medicine, Wayne State University, Detroit, MI, USA E-mail:kleerekm@trinity-health.org

\section{References}

1. McDougall D, Bhibhatbhan A, Nadeau J, Toth C. Adverse effects of corticosteroid therapy in neuromuscular diseased patients are common and receive insufficient prophylaxis. (This issue).

2. Ledwich LJ, Clarke K. Screening and treatment of glucocorticoid-induced osteoporosis in rheumatoid arthritis patients in an urban multispecialty practice. J Clin Rheum 2009; 15:61-4.

3. Lester Je, Dodwell D, Horsman JM, Mori S, Coleman Re. Current management of treatment-induced bone loss in women with breast cancer treated in the United Kingdom. Br J Cancer 2006;94:30-5.

4. Yee EF, White RE, Murata GH, Handanos C, Hoffman RM. Osteoporosis management in prostate cancer patients treated with androgen deprivation therapy. J Gen Intern Med 2007;22:1305-10. 